\title{
Pacific
}

Journal of

Mathematics

\section{VECTOR BUNDLES OVER A REAL ELLIPTIC CURVE}

INDRANIL BISWAS AND FLORENT SCHAFFHAUSER 


\title{
VECTOR BUNDLES OVER A REAL ELLIPTIC CURVE
}

\author{
INDRANIL BISWAS AND FLORENT SCHAFFHAUSER
}

\begin{abstract}
Given a geometrically irreducible smooth projective curve of genus 1 defined over the field of real numbers, and a pair of integers $r$ and $d$, we determine the isomorphism class of the moduli space of semistable vector bundles of rank $r$ and degree $d$ on the curve. When $r$ and $d$ are coprime, we describe the topology of the real locus and give a modular interpretation of its points. We also study, for arbitrary rank and degree, the moduli space of indecomposable vector bundles of rank $r$ and degree $d$, and determine its isomorphism class as a real algebraic variety.
\end{abstract}

\section{Introduction}

1A. Notation. In this paper, a real elliptic curve will be a triple $\left(X, x_{0}, \sigma\right)$ where $\left(X, x_{0}\right)$ is a complex elliptic curve (i.e., a compact connected Riemann surface of genus 1 with a marked point $x_{0}$ ) and $\sigma: X \rightarrow X$ is an antiholomorphic involution (also called a real structure). We do not assume that $x_{0}$ is fixed under $\sigma$. In particular, $X^{\sigma}:=\operatorname{Fix}(\sigma)$ is allowed to be empty.

The ged of two integers $r$ and $d$ will be denoted by $r \wedge d$.

In the introduction, we omit the definitions of stability and semistability of vector bundles, as well as that of real and quaternionic structures; all these definitions will be recalled in Section 2 .

1B. The case of genus zero. Vector bundles over a real Riemann surface of genus $g \geq 2$ have been studied from various points of view in the past few years: moduli spaces of real and quaternionic vector bundles were introduced through gaugetheoretic techniques in [Biswas et al. 2010], then related to the real points of the usual moduli variety in [Schaffhauser 2012]. In genus 0 , there are, up to isomorphism, only two possible real Riemann surfaces: the only compact Riemann surface of genus 0 is the Riemann sphere $\mathbb{C P}^{1}$ and it can be endowed either with the real structure $\left[z_{1}: z_{2}\right] \mapsto\left[\bar{z}_{1}: \bar{z}_{2}\right]$ or with the real structure $\left[z_{1}: z_{2}\right] \mapsto\left[-\bar{z}_{2}: \bar{z}_{1}\right]$. The real locus of the first real structure is $\mathbb{R} \mathbb{P}^{1}$ while the real locus of the second one is empty. Now, over $\mathbb{C P}^{1}$, two holomorphic line bundles are isomorphic if and

MSC2010: 14H52, 14H60, 14P25.

Keywords: Elliptic curves, vector bundles on curves, real algebraic varieties. 
only if they have the same degree and, by a theorem due to Grothendieck [1957], any holomorphic vector bundle over the Riemann sphere is isomorphic to a direct sum of line bundles. So, over $\mathbb{C P}^{1}$, the only stable vector bundles are the line bundles, a semistable vector bundle is necessarily polystable, and any vector bundle is isomorphic to a direct sum of semistable vector bundles, distinguished by their respective slopes and ranks. In particular, if $\mathcal{E}$ is semistable of rank $r$ and degree $d$, then $r$ divides $d$ and

$$
\mathcal{E} \simeq \mathcal{O}(d / r) \oplus \cdots \oplus \mathcal{O}(d / r)
$$

where $\mathcal{O}(1)$ is the positive degree generator of the Picard group of $\mathbb{C P}^{1}$ and $\mathcal{O}(k)$ is its $k$-th tensor power. This means that the moduli space of semistable vector bundles of rank $r$ and degree $d$ over $\mathbb{C P}^{1}$ is

$$
\mathcal{M}_{\mathbb{C P} 1}(r, d)=\left\{\begin{array}{cl}
\{\mathrm{pt}\} & \text { if } r \mid d, \\
\varnothing & \text { if } r \nmid d .
\end{array}\right.
$$

Assume now that a real structure $\sigma$ has been given on $\mathbb{C P}^{1}$. Then, if $\mathcal{L}$ is a holomorphic line bundle of degree $d$ over $\mathbb{C P}^{1}$, it is isomorphic to its Galois conjugate $\overline{\sigma^{*} \mathcal{L}}$, since they have the same degree. This implies that $\mathcal{L}$ is either real or quaternionic. Moreover, this real or quaternionic structure is unique up to real or quaternionic isomorphism, respectively; see Proposition 2.10. If the real structure $\sigma$ has real points, then quaternionic bundles must have even rank. Thus, when $\operatorname{Fix}(\sigma) \neq \varnothing$ in $\mathbb{C P}^{1}$, any line bundle (more generally, any direct sum of holomorphic line bundles) admits a canonical real structure. Of course, given a real vector bundle of the form $(\mathcal{L} \oplus \mathcal{L}, \tau \oplus \tau)$, where $\tau$ is a real structure on the line bundle $\mathcal{L}$, one can also construct the quaternionic structure

$$
\left(\begin{array}{cc}
0 & -\tau \\
\tau & 0
\end{array}\right)
$$

on $\mathcal{L} \oplus \mathcal{L}$. Note that the real vector bundle

$$
\left(\mathcal{L} \oplus \mathcal{L},\left(\begin{array}{ll}
0 & \tau \\
\tau & 0
\end{array}\right)\right)
$$

is isomorphic to $(\mathcal{L} \oplus \mathcal{L}, \tau \oplus \tau)$. When $\mathbb{C P}^{1}$ is equipped with its real structure with no real points, a given line bundle $\mathcal{L}$ of degree $k$ is again necessarily self-conjugate, so it has to be either real or quaternionic, but now real line bundles must have even degree and quaternionic line bundles must have odd degree [Biswas et al. 2010], so $\mathcal{L}$ admits a canonical real structure if $k$ is even and a canonical quaternionic structure if $k$ is odd. Consequently, when $\operatorname{Fix}(\sigma)=\varnothing$ in $\mathbb{C P}^{1}$, semistable holomorphic vector bundles of rank $r$ and degree $d=r k$ over $\mathbb{C P}^{1}$ admit a canonical real structure if $k$ is even and a canonical quaternionic structure if $k$ is odd. 
1C. Description of the results. The goal of the present paper is to analyze that same situation in the case of real Riemann surfaces of genus one. In particular, we completely identify the moduli space of semistable holomorphic vector bundles of rank $r$ and degree $d$ as a real algebraic variety (Theorem 1.1 below). Our main references are [Atiyah 1957b; Tu 1993]. In what follows, we denote by $\left(X, x_{0}\right)$ a complex elliptic curve and by $\mathcal{M}_{X}(r, d)$ the moduli space of semistable vector bundles of rank $r$ and degree $d$ over $X$, i.e., the set of $S$-equivalence classes of semistable holomorphic vector bundles of rank $r$ and degree $d$ over $X$ [Seshadri 1967]. Since $\left(X, x_{0}\right)$ is an elliptic curve, the results of Atiyah show that any holomorphic vector bundle on $X$ is again (as in genus 0 ) a direct sum of semistable vector bundles (see Theorem 3.3) but now there can be semistable vector bundles which are not polystable (see (3-2)) and also there can be stable vector bundles of rank higher than 1. Moreover, the moduli space $\mathcal{M}_{X}(r, d)$ is a nonsingular complex algebraic variety of dimension $h:=r \wedge d$. As a matter of fact, $\mathcal{M}_{X}(r, d)$ is isomorphic, as a complex algebraic variety, to the $(r \wedge d)$-fold symmetric product $\operatorname{Sym}^{r \wedge d}(X)$ of the complex elliptic curve $X$, and it contains stable bundles if and only if $r \wedge d=1$, in which case all semistable bundles are in fact stable. Let now $\sigma: X \rightarrow X$ be a real structure on $X$ (recall that the marked point $x_{0}$ is not assumed to be fixed under $\sigma$ ). Then the map $\mathcal{E} \mapsto \overline{\sigma^{*} \mathcal{E}}$ induces a real structure, again denoted by $\sigma$, on $\mathcal{M}_{X}(r, d)$, since it preserves the rank, the degree, and the $S$-equivalence class of semistable vector bundles [Schaffhauser 2012]. Our main result is then the following, to be proved in Section 2C.

Theorem 1.1. Let $h:=r \wedge d$. Then, as a real algebraic variety,

$$
\mathcal{M}_{X}(r, d) \simeq_{\mathbb{R}} \begin{cases}\operatorname{Sym}^{h}(X) & \text { if } X^{\sigma} \neq \varnothing, \\ \operatorname{Sym}^{h}(X) & \text { if } X^{\sigma}=\varnothing \text { and } d / h \text { is odd } \\ \operatorname{Sym}^{h}\left(\operatorname{Pic}_{X}^{0}\right) & \text { if } X^{\sigma}=\varnothing \text { and } d / h \text { is even. }\end{cases}
$$

We recall that $\operatorname{Pic}_{X}^{0}$ is isomorphic to $X$ over $\mathbb{C}$ (via the choice of $x_{0}$ ) but not over $\mathbb{R}$ when $X^{\sigma}=\varnothing$ because $\operatorname{Pic}_{X}^{0}$ has the real point corresponding to the trivial line bundle. In contrast, $\mathrm{Pic}_{X}^{1}$ is always isomorphic to $X$ over $\mathbb{R}$, as we shall recall in Section 2A. For any $d \in \mathbb{Z}$, the real structure of $\operatorname{Pic}_{X}^{d}$ is induced by the map $\mathcal{L} \mapsto \overline{\sigma^{*} \mathcal{L}}$, while the real structure of the $h$-fold symmetric product $\operatorname{Sym}^{h}(Y)$ of a real variety $(Y, \sigma)$ is induced by that of $Y$ in the following way: $\left[y_{1}, \ldots, y_{h}\right] \mapsto\left[\sigma\left(y_{1}\right), \ldots, \sigma\left(y_{h}\right)\right]$. Note that, if $r \wedge d=1$, then by Theorem 1.1 we have $\mathcal{M}_{X}(r, d) \simeq_{\mathbb{R}} X$ if $X^{\sigma} \neq \varnothing$ or $d$ is odd, and $\mathcal{M}_{X}(r, d) \simeq_{\mathbb{R}} \operatorname{Pic}_{X}^{0}$ if $X^{\sigma}=\varnothing$ and $d$ is even. This will eventually imply the following results on the topology and modular interpretation of the set of real points of $\mathcal{M}_{X}(r, d)$, analogous to those of [Schaffhauser 2012] for real curves of genus $g \geq 2$ (see Section 2D for a proof of Theorem 1.2; we point out that it will only be valid under the assumption that $r \wedge d=1$, in which case all 
semistable bundles are in fact stable; in particular a real point of $\mathcal{M}_{X}(r, d)$ is given by either a real bundle or a quaternionic bundle, in an essentially unique way; see Proposition 2.10).

Theorem 1.2. Assume that $r \wedge d=1$.

(1) If $X^{\sigma} \neq \varnothing$, then $\mathcal{M}_{X}(r, d)^{\sigma} \simeq X^{\sigma}$ has either 1 or 2 connected components. Points in either component correspond to real isomorphism classes of real vector bundles of rank $r$ and degree $d$ over $(X, \sigma)$, and two such bundles $\left(\mathcal{E}_{1}, \tau_{1}\right)$ and $\left(\mathcal{E}_{2}, \tau_{2}\right)$ lie in the same connected component if and only if $w_{1}\left(\mathcal{E}^{\tau_{1}}\right)=w_{1}\left(\mathcal{E}_{2}^{\tau_{2}}\right)$.

(2) If $X^{\sigma}=\varnothing$ and $d=2 e+1$, then $\mathcal{M}_{X}(r, 2 e+1)^{\sigma} \simeq X^{\sigma}$ is empty.

(3) If $X^{\sigma}=\varnothing$ and $d=2 e$, then $\mathcal{M}_{X}(r, 2 e)^{\sigma} \simeq\left(\operatorname{Pic}_{X}^{0}\right)^{\sigma}$ has two connected components, one consisting of real isomorphism classes of real bundles, the other consisting of quaternionic isomorphism classes of quaternionic bundles. These two components become diffeomorphic under the operation of tensoring a given bundle by a quaternionic line bundle of degree 0 .

In cases (1) and (3), each connected component of $\mathcal{M}_{X}(r, d)^{\sigma}$ is diffeomorphic to $S^{1}$.

In particular, the formulae of Liu and Schaffhauser [2013] (see also [Baird 2014]), giving the mod 2 Betti numbers of the connected components of $\mathcal{M}_{X}(r, d)^{\sigma}$ when $r \wedge d=1$ are still valid for $g=1$. In contrast, when $r \wedge d \neq 1$, the formulae do not seem to be interpretable in any way since, over an elliptic curve, the dimension of $\mathcal{M}_{X}(r, d)$ is $r \wedge d$, not $r^{2}(g-1)+1$.

In the third and final section of the paper, we investigate the properties of indecomposable vector bundles over real elliptic curves. Recall that a holomorphic vector bundle $\mathcal{E}$ over a complex curve $X$ is said to be indecomposable if it is not isomorphic to a direct sum of nontrivial holomorphic bundles. When $X$ is of genus 1 , there exists a moduli variety $\mathcal{I}_{X}(r, d)$ whose points are isomorphism classes of indecomposable vector bundles of rank $r$ and degree $d$ : it was constructed by Atiyah [1957b] and revisited by Tu [1993], as will be recalled in Theorems 3.2 and 3.4. We will then see in Section 3C that we can extend their approach to the case of real elliptic curves and obtain the following characterization of $\mathcal{I}_{X}(r, d)$ as a real algebraic variety.

Theorem 1.3. Let $\left(X, x_{0}, \sigma\right)$ be a real elliptic curve. Let $\mathcal{I}_{X}(r, d)$ be the set of isomorphism classes of indecomposable vector bundles of rank $r$ and degree $d$ and set $h:=r \wedge d, r^{\prime}:=r / h, d^{\prime}:=d / h$. Then

$$
\mathcal{I}_{X}(r, d) \simeq_{\mathbb{R}} \mathcal{M}_{X}\left(r^{\prime}, d^{\prime}\right) \simeq_{\mathbb{R}}\left\{\begin{array}{cl}
X & \text { if } X^{\sigma} \neq \varnothing, \\
X & \text { if } X^{\sigma}=\varnothing \text { and } d^{\prime} \text { is odd }, \\
\operatorname{Pic}_{X}^{0} & \text { if } X^{\sigma}=\varnothing \text { and } d^{\prime} \text { is even. }
\end{array}\right.
$$


By combining Theorems 1.2 and 1.3, we obtain the following topological description of the set of real points of $\mathcal{I}_{X}(r, d)$, valid even when $r \wedge d \neq 1$.

Theorem 1.4. Denote by $\mathcal{I}_{X}(r, d)^{\sigma}$ the fixed points of the real structure $\mathcal{E} \mapsto \overline{\sigma^{*} \mathcal{E}}$ in $\mathcal{I}_{X}(r, d)$.

(1) If $X^{\sigma} \neq \varnothing$, then $\mathcal{I}_{X}(r, d)^{\sigma} \simeq X^{\sigma}$ consists of real isomorphism classes of real and indecomposable vector bundles of rank $r$ and degree $d$. It has either one or two connected components, according to whether $X^{\sigma}$ has one or two connected components, and these are distinguished by the Stiefel-Whitney classes of the real parts of the real bundles that they contain.

(2) If $X^{\sigma}=\varnothing$ and $d /(r \wedge d)=2 e+1$, then $\mathcal{I}_{X}(r, d)^{\sigma} \simeq X^{\sigma}$ is empty.

(3) If $X^{\sigma}=\varnothing$ and $d /(r \wedge d)=2 e$, then $\mathcal{I}_{X}(r, d)^{\sigma} \simeq\left(\operatorname{Pic}_{X}^{0}\right)^{\sigma}$ has two connected components, one consisting of real isomorphism classes of vector bundles which are both real and indecomposable and one consisting of quaternionic isomorphism classes of vector bundles which are both quaternionic and indecomposable. These two components become diffeomorphic under the operation of tensoring a given bundle by a quaternionic line bundle of degree 0 .

In cases (1) and (3), each connected component of the set of real points of $\mathcal{I}_{X}(r, d)$ is diffeomorphic to $S^{1}$.

\section{Moduli spaces of semistable vector bundles over an elliptic curve}

2A. Real elliptic curves and their Picard varieties. The real points of Picard varieties of real algebraic curves were studied, for instance, by Gross and Harris [1981]. We summarize here some of their results, specializing to the case of genus 1 curves.

Let $X$ be a compact connected Riemann surface of genus 1 . To each point $x \in X$, there is associated a holomorphic line bundle $\mathcal{L}(x)$, of degree 1 , whose holomorphic sections have a zero of order 1 at $x$ and no other zeros or poles. Since $X$ is compact, the map $X \rightarrow \operatorname{Pic}_{X}^{1}$ thus defined, called the Abel-Jacobi map, is injective. And since $X$ has genus 1 , it is also surjective. The choice of a point $x_{0} \in X$ defines an isomorphism $\operatorname{Pic}_{X}^{0} \stackrel{\sim}{\longrightarrow} \operatorname{Pic}_{X}^{1}$, obtained by tensoring by $\mathcal{L}\left(x_{0}\right)$. In particular, $X \simeq \operatorname{Pic}_{X}^{1}$ is isomorphic to $\operatorname{Pic}_{X}^{0}$ as a complex analytic manifold and inherits, moreover, a structure of Abelian group with $x_{0}$ as the neutral element.

If $\sigma: X \rightarrow X$ is a real structure on $X$, the Picard variety $\operatorname{Pic}_{X}^{d}$, whose points represent isomorphism classes of holomorphic line bundles of degree $d$, has a canonical real structure, defined by $\mathcal{L} \mapsto \overline{\sigma^{*} \mathcal{L}}$ (observe that this antiholomorphic involution, which we will still denote by $\sigma$, indeed preserves the degree). Since $\mathcal{L}(\sigma(x)) \simeq \overline{\sigma^{*}(\mathcal{L}(x))}$, the Abel-Jacobi map $X \rightarrow \operatorname{Pic}_{X}^{1}$ is defined over $\mathbb{R}$, meaning that it commutes with the real structures of $X$ and $\mathrm{Pic}_{X}^{1}$. We also call such a map a real map. If $X^{\sigma} \neq \varnothing$, we can choose $x_{0} \in X^{\sigma}$ and then $\mathcal{L}\left(x_{0}\right)$ will satisfy 
$\overline{\sigma^{*} \mathcal{L}\left(x_{0}\right)} \simeq \mathcal{L}\left(x_{0}\right)$ so the isomorphism $\operatorname{Pic}_{X}^{0} \stackrel{\sim}{\longrightarrow} \operatorname{Pic}_{X}^{1}$ obtained by tensoring by $\mathcal{L}\left(x_{0}\right)$ will also be defined over $\mathbb{R}$. More generally, by tensoring by a suitable power of $\mathcal{L}\left(x_{0}\right)$, we obtain real isomorphisms $\operatorname{Pic}_{X}^{d} \simeq \operatorname{Pic}_{X}^{1}$ for any $d \in \mathbb{Z}$. If now $X^{\sigma}=\varnothing$, then we actually cannot choose $x_{0}$ in such a way that $\mathcal{L}\left(\sigma\left(x_{0}\right)\right) \simeq \mathcal{L}\left(x_{0}\right)$ - see [Gross and Harris 1981] or Theorem 2.8 below; the reason is that such a line bundle would be either real or quaternionic but, over a real curve of genus 1 with no real points, real and quaternionic line bundles must have even degree - but we may consider the holomorphic line bundle of degree 2 defined by the divisor $x_{0}+\sigma\left(x_{0}\right)$, call it $\mathcal{L}$, say. Then $\overline{\sigma^{*} \mathcal{L}} \simeq \mathcal{L}$ and, by tensoring by an appropriate tensor power of it, we have the real isomorphisms

$$
\operatorname{Pic}_{X}^{d} \simeq_{\mathbb{R}} \begin{cases}\operatorname{Pic}_{X}^{1} & \text { if } d=2 e+1, \\ \operatorname{Pic}_{X}^{0} & \text { if } d=2 e .\end{cases}
$$

So, when the genus of $X$ is 1 , we have the following result:

Theorem 2.1. Let $\left(X, x_{0}, \sigma\right)$ be a real elliptic curve.

(1) If $X^{\sigma} \neq \varnothing$, then for all $d \in \mathbb{Z}$,

$$
\operatorname{Pic}_{X}^{d} \simeq_{\mathbb{R}} X
$$

(2) If $X^{\sigma}=\varnothing$, then

$$
\operatorname{Pic}_{X}^{d} \simeq_{\mathbb{R}}\left\{\begin{array}{cl}
X & \text { if } d=2 e+1, \\
\operatorname{Pic}_{X}^{0} & \text { if } d=2 e .
\end{array}\right.
$$

2B. Semistable vector bundles. Let $X$ be a compact connected Riemann surface of genus $g$ and recall that the slope of a nonzero holomorphic vector bundle $\mathcal{E}$ on $X$ is by definition the ratio $\mu(\mathcal{E})=\operatorname{deg}(\mathcal{E}) / \operatorname{rk}(\mathcal{E})$ of its degree by its rank. The vector bundle $\mathcal{E}$ is called stable if for any nonzero proper subbundle $\mathcal{F} \subset \mathcal{E}$, one has $\mu(\mathcal{F})<\mu(\mathcal{E})$. Analogously, $\mathcal{E}$ is called semistable if $\mu(\mathcal{F}) \leq \mu(\mathcal{E})$. By a theorem of Seshadri [1967], any semistable vector bundle $\mathcal{E}$ of rank $r$ and degree $d$ admits a filtration whose successive quotients are stable bundles of the same slope, necessarily equal to $d / r$. Such a filtration, called a Jordan-Hölder filtration, is not unique but the graded objects associated to any two such filtrations are isomorphic. The isomorphism class thus defined is denoted by $\operatorname{gr}(\mathcal{E})$ and holomorphic vector bundles which are isomorphic to direct sums of stable vector bundles of equal slope are called polystable vector bundles. Moreover, two semistable vector bundles $\mathcal{E}_{1}$ and $\mathcal{E}_{2}$ are called $S$-equivalent if $\operatorname{gr}\left(\mathcal{E}_{1}\right)=\operatorname{gr}\left(\mathcal{E}_{2}\right)$ and Seshadri proved in [Seshadri 1967] that, when $g \geq 2$, the set of $S$-equivalence classes of semistable vector bundles of rank $r$ and degree $d$ admits a structure of complex projective variety of dimension $r^{2}(g-1)+1$ and is nonsingular when $r \wedge d=1$ but usually singular when $r \wedge d \neq 1$ (unless, in fact, $g=2, r=2$, and $d=0$ ). Finally, when $g \geq 2$, there are always stable bundles of rank $r$ and degree $d$ over $X$ [Narasimhan and Seshadri 1965]. 
These come from irreducible rank $r$ unitary representations of a certain central extension of $\pi_{1}(X)$ by $\mathbb{Z}$, determined by $d$ up to isomorphism). If now $g=1$, then the results of Atiyah [1957b] and Tu [1993] show that the set of $S$-equivalence classes of semistable vector bundles of rank $r$ and degree $d$ admits a structure of nonsingular complex projective variety of dimension $r \wedge d$ (which is consistent with the formula for $g \geq 2$ only when $r$ and $d$ are coprime). But now stable vector bundles of rank $r$ and degree $d$ can only exist if $r \wedge d=1$, as Tu showed [1993, Theorem A] following Atiyah's results. In particular, the structure of polystable vector bundles over a complex elliptic curve is rather special, as recalled next.

Proposition 2.2 (Atiyah-Tu). Let $\mathcal{E}$ be a polystable holomorphic vector bundle of rank $r$ and degree $d$ over a compact connected Riemann surface $X$ of genus 1 . Set $h:=r \wedge d, r^{\prime}:=r / h$, and $d^{\prime}:=d / h$. Then $\mathcal{E} \simeq \mathcal{E}_{1} \oplus \cdots \oplus \mathcal{E}_{h}$ where each $\mathcal{E}_{i}$ is a stable holomorphic vector bundle of rank $r^{\prime}$ and degree $d^{\prime}$.

Proof. By definition, a polystable bundle of rank $r$ and degree $d$ is isomorphic to a direct sum $\mathcal{E}_{1} \oplus \cdots \oplus \mathcal{E}_{k}$ of stable bundles of slope $d / r=d^{\prime} / r^{\prime}$. Since $d^{\prime} \wedge r^{\prime}=1$ and each $\mathcal{E}_{i}$ is stable of slope $d^{\prime} / r^{\prime}$, each $\mathcal{E}_{i}$ must have rank $r^{\prime}$ and degree $d^{\prime}$ (because stable bundles over elliptic curves must have coprime rank and degree). Since $\operatorname{rk}\left(\mathcal{E}_{1} \oplus \cdots \oplus \mathcal{E}_{k}\right)=k r^{\prime}=\operatorname{rk}(\mathcal{E})=r$, we have indeed $k=h$.

To understand the moduli space $\mathcal{M}_{X}(r, d)$ of semistable holomorphic vector bundles of rank $r$ and degree $d$ over a complex elliptic curve $X$, one then has the next two theorems.

Theorem 2.3 (Atiyah-Tu). Let $X$ be a compact connected Riemann surface of genus 1 and assume that $r \wedge d=1$. Then the determinant map det: $\mathcal{M}_{X}(r, d) \rightarrow \operatorname{Pic}_{X}^{d}$ is an isomorphism of complex analytic manifolds of dimension 1.

Note that, when $r \wedge d=1$, any semistable vector bundle of rank $r$ and degree $d$ is in fact stable (over a curve of arbitrary genus) and that, to prove Theorem 2.3, it is in particular necessary to show that a stable vector bundle $\mathcal{E}$ of rank $r$ and degree $d$ over a complex elliptic curve $X$ satisfies $\mathcal{E} \otimes \mathcal{L} \simeq \mathcal{E}$ if and only if $\mathcal{L}$ is an $r$-torsion point in $\operatorname{Pic}_{X}^{0}$ (i.e., $\mathcal{L}^{\otimes r} \simeq \mathcal{O}_{X}$ ), a phenomenon which only occurs in genus 1 .

If now $h:=r \wedge d \geq 2$, then we know, by Proposition 2.2, that a semistable vector bundle of rank $r$ and degree $d$ is isomorphic to the direct sum of $h$ stable vector bundles of rank $r^{\prime}=r / h$ and degree $d^{\prime}=d / h$. Combining this with Theorem 2.3, one obtains:

Theorem 2.4 [Tu 1993, Theorem 1]. Let X be a compact connected Riemann surface of genus 1 and denote by $h:=r \wedge d$. Then there is an isomorphism of complex analytic manifolds:

$$
\mathcal{M}_{X}(r, d) \stackrel{\sim}{\longrightarrow} \operatorname{Sym}^{h}\left(\operatorname{Pic}_{X}^{d / h}\right), \quad \mathcal{E} \simeq \mathcal{E}_{1} \oplus \cdots \oplus \mathcal{E}_{h} \mapsto\left[\operatorname{det} \mathcal{E}_{i}\right]_{1 \leq i \leq h} .
$$


In particular, $\mathcal{M}_{X}(r, d)$ has dimension $h=r \wedge d$. Since the choice of a point $x_{0} \in X$ provides an isomorphism $\operatorname{Pic}_{X}^{d} \simeq_{\mathbb{C}} X$, we have indeed $\mathcal{M}_{X}(r, d) \simeq_{\mathbb{C}} \operatorname{Sym}^{h}(X)$. In the next section, we will analyze the corresponding situation over $\mathbb{R}$. But first we recall the basics about real and quaternionic vector bundles.

Let $(X, \sigma)$ be a real Riemann surface, i.e., a Riemann surface $X$ endowed with a real structure $\sigma$. A real holomorphic vector bundle over $(X, \sigma)$ is a pair $(\mathcal{E}, \tau)$ such that $\mathcal{E} \rightarrow X$ is a holomorphic vector bundle over $X$ and $\tau: \mathcal{E} \rightarrow \mathcal{E}$ is an antiholomorphic map such that

(1) the diagram

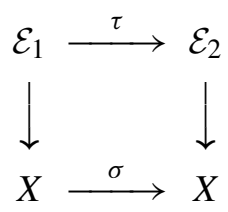

is commutative;

(2) the map $\tau$ is fiberwise $\mathbb{C}$-antilinear: $\forall v \in \mathcal{E}, \forall \lambda \in \mathbb{C}, \tau(\lambda v)=\bar{\lambda}(v)$;

(3) $\tau^{2}=\operatorname{Id}_{\mathcal{E}}$.

A quaternionic holomorphic vector bundle over $(X, \sigma)$ is a pair $(\mathcal{E}, \tau)$ satisfying conditions (1) and (2) above, as well as a modified third condition:

$\left(3^{\prime}\right) \tau^{2}=-\operatorname{Id}_{\mathcal{E}}$.

A homomorphism $\varphi:\left(\mathcal{E}_{1}, \tau_{1}\right) \rightarrow\left(\mathcal{E}_{2}, \tau_{2}\right)$ between two real or quaternionic vector bundles is a holomorphic map $\varphi: \mathcal{E}_{1} \rightarrow \mathcal{E}_{2}$ such that

(1) the diagram

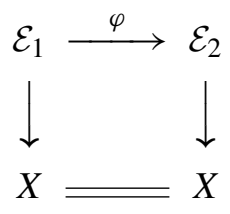

is commutative;

(2) $\varphi \circ \tau_{1}=\tau_{2} \circ \varphi$.

A real or quaternionic holomorphic vector bundle is called stable if for any $\tau$-invariant subbundle $\mathcal{F} \subset \mathcal{E}$, one has $\mu(\mathcal{F})<\mu(\mathcal{E})$. It is called semistable if for any such $\mathcal{F}$, one has $\mu(\mathcal{F}) \leq \mu(\mathcal{F})$. As shown in [Schaffhauser 2012], $(\mathcal{E}, \tau)$ is semistable as a real vector bundle if and only $\mathcal{E}$ is semistable as a holomorphic vector bundle but $(\mathcal{E}, \tau)$ may be stable as a real vector bundle while being only polystable as a holomorphic vector bundle (when $\mathcal{E}$ is in fact stable, we will say that $(\mathcal{E}, \tau)$ is geometrically stable). However, any semistable real vector bundle admits real Jordan-Hölder filtrations (where the successive quotients can sometimes be stable in the real sense only) and there is a corresponding notion of polystable 
real vector bundle, which turns out to be equivalent to being polystable and real. Analogous results hold for quaternionic vector bundles. Real and quaternionic vector bundles over a compact connected real Riemann surface $(X, \sigma)$ were topologically classified in [Biswas et al. 2010]. If $X^{\sigma} \neq \varnothing$, a real vector bundle $(\mathcal{E}, \tau)$ over $(X, \sigma)$ defines in particular a real vector bundle in the ordinary sense $\mathcal{E}^{\tau} \rightarrow X^{\sigma}$; hence an associated first Stiefel-Whitney class $w_{1}\left(\mathcal{E}^{\tau}\right) \in H^{1}\left(X^{\sigma} ; \mathbb{Z} / 2 \mathbb{Z}\right) \simeq(\mathbb{Z} / 2 \mathbb{Z})^{n}$, where $n \in\{0, \ldots, g+1\}$ is the number of connected components of $X^{\sigma}$. The topological classification of real and quaternionic vector bundles then goes as follows.

Theorem 2.5 [Biswas et al. 2010]. Let $(X, \sigma)$ be a compact connected real Riemann surface.

(1) If $X^{\sigma} \neq \varnothing$, real vector bundles over $(X, \sigma)$ are classified up to smooth isomorphism by the numbers $r=\operatorname{rk}(\mathcal{E}), d=\operatorname{deg}(\mathcal{E})$, and $\left(s_{1}, \ldots, s_{n}\right)=w_{1}\left(\mathcal{E}^{\tau}\right)$, subject to the condition $s_{1}+\cdots+s_{n} \equiv d(\bmod 2)$. Quaternionic vector bundles must have even rank and degree in this case and are classified up to smooth isomorphism by the pair $(2 r, 2 d)$.

(2) If $X^{\sigma}=\varnothing$, real vector bundles over $(X, \sigma)$ must have even degree and are classified up to smooth isomorphism by the pair $(r, 2 d)$. Quaternionic vector bundles are classified up to smooth isomorphism by the pair $(r, d)$, subject to the condition $d+r(g-1) \equiv 0(\bmod 2)$. In particular, if $g=1$, real and quaternionic vector bundles alike must have even degree.

Theorem 2.5 will be useful in Section 2D, for the proof of Theorem 1.2.

2C. The real structure of the moduli space. Let first $(X, \sigma)$ be a real Riemann surface of arbitrary genus $g$. Then the involution $\mathcal{E} \mapsto \overline{\sigma^{*} \mathcal{E}}$ preserves the rank and the degree of a holomorphic vector bundle and the bundle $\overline{\sigma^{*} \mathcal{E}}$ is stable if and only if $\mathcal{E}$ is. The analogous statement holds for semistable bundles. Moreover, if $\mathcal{E}$ is semistable, a Jordan-Hölder filtration of $\mathcal{E}$ is mapped to a Jordan-Hölder filtration of $\overline{\sigma^{*} \mathcal{E}}$, so, for any $g$, the moduli space $\mathcal{M}_{X}(r, d)$ of semistable holomorphic vector bundles of rank $r$ and degree $d$ on $X$ has an induced real structure. Assume now that $g=1$ and let us prove Theorem 1.1.

Proof of Theorem 1.1. Since, for any vector bundle $\mathcal{E}$ one has $\operatorname{det}\left(\overline{\sigma^{*} \mathcal{E}}\right)=\overline{\sigma^{*}(\operatorname{det} \mathcal{E})}$, the map

$$
\mathcal{M}_{X}(r, d) \stackrel{\sim}{\longrightarrow} \operatorname{Sym}^{h}\left(\operatorname{Pic}_{X}^{d / h}\right), \quad \mathcal{E} \simeq \mathcal{E}_{1} \oplus \cdots \oplus \mathcal{E}_{h} \mapsto\left[\operatorname{det} \mathcal{E}_{i}\right]_{1 \leq i \leq h}
$$

of Theorem 2.4 is a real map. If $X^{\sigma} \neq \varnothing$, then by Theorem 2.1 we have

$$
\operatorname{Pic}_{X}^{d} \simeq_{\mathbb{R}} \operatorname{Pic}_{X}^{0} \simeq_{\mathbb{R}} X
$$

so $\mathcal{M}_{X}(r, d) \simeq_{\mathbb{R}} \operatorname{Sym}^{h}(X)$ in this case. And if $X^{\sigma}=\varnothing$, we distinguish between the 
cases $d=2 e+1$ and $d=2 e$ to obtain, again by Theorem 2.1, that

$$
\mathcal{M}_{X}(r, d) \simeq_{\mathbb{R}} \begin{cases}\operatorname{Sym}^{h}(X) & \text { if } d / h \text { is odd, } \\ \operatorname{Sym}^{h}\left(\operatorname{Pic}_{X}^{0}\right) & \text { if } d / h \text { is even, }\end{cases}
$$

which finishes the proof of Theorem 1.1.

Let us now focus on the case $d=0$, where there is a nice alternate description of the moduli variety in terms of representations of the fundamental group of the elliptic curve $\left(X, x_{0}\right)$. Since $\pi_{1}\left(X, x_{0}\right) \simeq \mathbb{Z}^{2}$ is a free Abelian group on two generators, a rank $r$ unitary representation of it is entirely determined by the data of two commuting unitary matrices $u_{1}, u_{2}$ in $\boldsymbol{U}(r)$ (in particular, such a representation is never irreducible unless $r=1$ ), and we may assume that these two matrices lie in the maximal torus $\boldsymbol{T}_{r} \subset \boldsymbol{U}(r)$ consisting of diagonal unitary matrices. The Weyl group of $\boldsymbol{T}_{r}$ is $\boldsymbol{W}_{r} \simeq \mathfrak{S}_{r}$, the symmetric group on $r$ letters, and one has

$$
\operatorname{Hom}\left(\pi_{1}\left(X, x_{0}\right) ; \boldsymbol{U}(r)\right) / \boldsymbol{U}(r) \simeq \operatorname{Hom}\left(\pi_{1}\left(X, x_{0}\right) ; \boldsymbol{T}_{r}\right) / \boldsymbol{W}_{r} .
$$

Note that since $\pi_{1}\left(X, x_{0}\right)$ is Abelian, there is a well-defined action of $\sigma$ on it even if $x_{0} \notin X^{\sigma}$ : a loop $\gamma$ at $x_{0}$ is sent to the loop $\sigma \circ \gamma$ at $\sigma\left(x_{0}\right)$ then brought back to $x_{0}$ by conjugation by an arbitrary path between $x_{0}$ and $\sigma\left(x_{0}\right)$. Combining this with the involution $u \mapsto \bar{u}$ of $\boldsymbol{U}(r)$, we obtain an action of $\sigma$ on $\operatorname{Hom}\left(\pi_{1}\left(X, x_{0}\right) ; \boldsymbol{U}(r)\right)$, defined by sending a representation $\rho$ to the representation $\sigma \rho \sigma$. This action preserves the subset $\operatorname{Hom}\left(\pi_{1}\left(X, x_{0}\right) ; \boldsymbol{T}_{r}\right)$ and is compatible with the conjugacy action of $\boldsymbol{U}(r)$ in the sense that $\sigma\left(\operatorname{Ad}_{u} \rho\right) \sigma=\operatorname{Ad}_{\sigma(u)}\left(\sigma \rho \sigma^{-1}\right)$, so it induces an involution on the representation varieties $\operatorname{Hom}\left(\pi_{1}\left(X, x_{0}\right) ; \boldsymbol{U}(r)\right) / \boldsymbol{U}(r)$ and $\operatorname{Hom}\left(\pi_{1}\left(X, x_{0}\right) ; \boldsymbol{T}_{r}\right) / \boldsymbol{W}_{r}$ and the bijection (2-1) is equivariant for the actions just described. By the results of Friedman, Morgan and Witten [Friedman et al. 1998] and Laszlo [1998], this representation variety is in fact isomorphic to the moduli space $\mathcal{M}_{X}(r, 0)$. Moreover, the involution $\mathcal{E} \mapsto \overline{\sigma^{*} \mathcal{E}}$ on bundles corresponds to the involution $\rho \mapsto \sigma \rho \sigma$ on unitary representations. Moreover,

$$
\boldsymbol{T}_{r} \simeq \underbrace{\boldsymbol{U}(1) \times \cdots \times \boldsymbol{U}(1)}_{r \text { times }} \simeq \boldsymbol{U}(1) \otimes_{\mathbb{Z}} \mathbb{Z}^{r}
$$

as Abelian Lie groups, where $\mathbb{Z}^{r}$ can be interpreted as $\pi_{1}\left(\boldsymbol{T}_{r}\right)$. In particular, the Galois action induced on $\mathbb{Z}^{r}$ by the complex conjugation on $\boldsymbol{T}_{r}$ is simply $\left(n_{1}, \ldots, n_{r}\right) \mapsto\left(-n_{1}, \ldots,-n_{r}\right)$ and the isomorphism $\boldsymbol{T}_{r} \simeq \boldsymbol{U}(1) \otimes \mathbb{Z}^{r}$ is equivariant with respect to these natural real structures. Finally, the bijection

$$
\operatorname{Hom}\left(\pi_{1}\left(X, x_{0}\right) ; \boldsymbol{T}_{r}\right) \simeq \operatorname{Hom}\left(\pi_{1}\left(X, x_{0}\right) ; \boldsymbol{U}(1)\right) \otimes \mathbb{Z}^{r}
$$

is also equivariant, and the representation variety $\operatorname{Hom}\left(\pi_{1}\left(X, x_{0}\right) ; \boldsymbol{U}(1)\right)$ is isomorphic to $\mathrm{Pic}_{X}^{0}$ as a real variety. We have thus proved the following result, which is an analogue over $\mathbb{R}$ of one of the results in [Friedman et al. 1998; Laszlo 1998]. 
Theorem 2.6. Let $\left(X, x_{0}, \sigma\right)$ be a real elliptic curve. Then the map

$$
\left(\mathrm{Pic}_{X}^{0} \otimes_{\mathbb{Z}} \mathbb{Z}^{r}\right) \simeq\left(\mathrm{Pic}_{X}^{0}\right)^{r} \rightarrow \mathcal{M}(r, 0), \quad\left(\mathcal{L}_{1}, \ldots, \mathcal{L}_{r}\right) \mapsto \mathcal{L}_{1} \oplus \cdots \oplus \mathcal{L}_{r}
$$

induces an isomorphism

$$
\mathcal{M}_{X}(r, 0) \simeq_{\mathbb{R}}\left(\operatorname{Pic}_{X}^{0} \otimes \mathbb{Z}^{r}\right) / \mathfrak{S}_{r},
$$

where the symmetric group $\mathfrak{S}_{r}$ acts on $\mathbb{Z}^{r}$ by permutation.

When $X^{\sigma} \neq \varnothing$, one can further identify $\operatorname{Pic}_{X}^{0}$ with $X$ over $\mathbb{R}$ and obtain the isomorphism

$$
\mathcal{M}_{X}(r, 0) \simeq_{\mathbb{R}}\left(X \otimes \mathbb{Z}^{r}\right) / \mathfrak{S}_{r} .
$$

The results of Section 3 will actually give an alternate proof of Theorem 2.6, by using the theory of indecomposable vector bundles over elliptic curves. We point out that algebraic varieties of the form $\left(X \otimes \pi_{1}(\boldsymbol{T})\right) / \boldsymbol{W}_{\boldsymbol{T}}$ for $X$ a complex elliptic curve have been studied for instance by Looijenga [1976], who identified them with certain weighted projective spaces determined by the root system of $\boldsymbol{T}$, when the ambient group $G \supset \boldsymbol{T}$ is semisimple. Theorem 2.6 shows that, over $\mathbb{R}$, it may sometimes be necessary to replace $X$ by $\operatorname{Pic}_{X}^{0}$.

To conclude on the case where $d=0$, we recall that, on $\mathcal{M}_{X}(r, 0)$, there exists another real structure, obtained from the real structure $\mathcal{E} \mapsto \overline{\sigma^{*} \mathcal{E}}$ by composing it with the holomorphic involution $\mathcal{E} \mapsto \mathcal{E}^{*}$, which in general sends a vector bundle of degree $d$ to a vector bundle of degree $-d$, so preserves only the moduli spaces $\mathcal{M}_{X}(r, 0)$. Denote by

$$
\eta_{r}: \mathcal{M}_{X}(r, 0) \rightarrow \mathcal{M}_{X}(r, 0), \quad \mathcal{E} \mapsto \bar{\sigma}^{*} \mathcal{E}^{*}
$$

this new real structure on the moduli space $\mathcal{M}_{X}(r, 0)$. In particular, we have

$$
\eta_{1}: \mathrm{Pic}_{X}^{0} \rightarrow \operatorname{Pic}_{X}^{0}, \quad \mathcal{L} \mapsto \bar{\sigma}^{*} \mathcal{L}^{*}
$$

and we note that $\eta_{1}$ has real points because it fixes the trivial line bundle. The real elliptic curve $\left(\operatorname{Pic}_{X}^{0}, \eta_{1}\right)$ is, in general, not isomorphic to $(X, \sigma)$, even when $\sigma$ has fixed points. We can nonetheless characterize the new real structure of the moduli spaces $\mathcal{M}_{X}(r, 0)$ in the following way:

Proposition 2.7. The real variety $\left(\mathcal{M}_{X}(r, 0), \eta_{r}\right)$ is isomorphic to the r-fold symmetric product of the real elliptic curve $\left(\mathrm{Pic}_{X}^{0}, \eta_{1}\right)$.

Proof. The proposition is proved in the same way as Theorem 2.6, changing only the real structures under consideration.

2D. Topology of the set of real points in the coprime case. In rank 1, the topology of the set of real points of $\mathrm{Pic}_{X}^{d}$ is well understood and so is the modular interpretation of its elements. 
Theorem 2.8 ([Gross and Harris 1981], case $g=1)$. Let $(X, \sigma)$ be a compact real Riemann surface of genus 1 and let $d \in \mathbb{Z}$.

(1) If $X^{\sigma} \neq \varnothing$, then $\left(\operatorname{Pic}_{X}^{d}\right)^{\sigma} \simeq X^{\sigma}$ has 1 or 2 connected components. Elements of $\left(\operatorname{Pic}_{X}^{d}\right)^{\sigma}$ correspond to real isomorphism classes of real holomorphic line bundles over $(X, \sigma)$ and two such real line bundles $\left(\mathcal{L}_{1}, \tau_{1}\right)$ and $\left(\mathcal{L}_{2}, \tau_{2}\right)$ lie in the same connected component of $\left(\operatorname{Pic}_{X}^{d}\right)^{\sigma}$ if and only if $w_{1}\left(\mathcal{L}_{1}^{\tau_{1}}\right)=w_{1}\left(\mathcal{L}_{2}^{\tau_{2}}\right)$.

(2) If $X^{\sigma}=\varnothing$ and $d=2 e+1$, then $\left(\operatorname{Pic}_{X}^{d}\right)^{\sigma} \simeq X^{\sigma}$ is empty.

(3) If $X^{\sigma}=\varnothing$ and $d=2 e$, then $\left(\mathrm{Pic}_{X}^{d}\right)^{\sigma} \simeq\left(\mathrm{Pic}_{X}^{0}\right)^{\sigma}$ has 2 connected components, corresponding to isomorphism classes of either real or quaternionic line bundles of degree $d$, depending on the connected component of $\left(\operatorname{Pic}_{X}^{d}\right)^{\sigma}$ in which they lie.

In cases (1) and (3), any given connected component of $\left(\mathrm{Pic}_{X}^{d}\right)^{\sigma}$ is diffeomorphic to $S^{1}$.

For real Riemann surfaces of genus $g \geq 2$, the topology of $\left(\operatorname{Pic}_{X}^{d}\right)^{\sigma}$, in particular the number of connected components, is a bit more involved but also covered in [Gross and Harris 1981], the point being that these components are indexed by the possible topological types of real and quaternionic line bundles over $(X, \sigma)$. For vector bundles of rank $r \geq 2$ on real Riemann surfaces of genus $g \geq 2$, a generalization of the results of Gross and Harris was obtained in [Schaffhauser 2012]: we recall here the result for coprime rank and degree (in general, a similar but more complicated result holds provided one restricts one's attention to the stable locus in $\left.\mathcal{M}_{X}(r, d)\right)$. The coprime case is the case that we will actually generalize to genus 1 curves (where stable bundles can only exist in coprime rank and degree).

Theorem 2.9 [Schaffhauser 2012]. Let $(X, \sigma)$ be a compact real Riemann surface of genus $g \geq 2$ and assume that $r \wedge d=1$. The number of connected component of $\mathcal{M}_{X}(r, d)^{\sigma}$ is equal to:

(1) $2^{n-1}$ if $X^{\sigma}$ has $n>0$ connected components. In this case, elements of $\mathcal{M}_{X}(r, d)^{\sigma}$ correspond to real isomorphism classes of real holomorphic vector bundles of rank $r$ and degree $d$ and two such bundles $\left(\mathcal{E}_{1}, \tau_{1}\right)$ and $\left(\mathcal{E}_{2}, \tau_{2}\right)$ lie in the same connected component if and only if $w_{1}\left(\mathcal{E}^{\tau_{1}}\right)=w_{1}\left(\mathcal{E}_{2}^{\tau_{2}}\right)$.

(2) 0 if $X^{\sigma}=\varnothing, d$ is odd and $r(g-1)$ is even.

(3) 1 if $X^{\sigma}=\varnothing, d$ is odd and $r(g-1)$ is odd, in which case the elements of $\mathcal{M}_{X}(r, d)^{\sigma}$ correspond to quaternionic isomorphism classes of quaternionic vector bundles of rank $r$ and degree $d$.

(4) 1 if $X^{\sigma}=\varnothing, d$ is even and $r(g-1)$ is odd, in which case the elements of $\mathcal{M}_{X}(r, d)^{\sigma}$ correspond to real isomorphism classes of real vector bundles of rank $r$ and degree $d$. 
(5) 2 if $X^{\sigma}=\varnothing, d$ is even and $r(g-1)$ is even, in which case there is one component consisting of real isomorphism classes of real vector bundles of rank $r$ and degree $d$ while the other consists of quaternionic isomorphism classes of quaternionic vector bundles of rank $r$ and degree $d$.

Now, using Theorem 1.1, we can extend Theorem 2.9 to the case $g=1$. Indeed, to prove Theorem 1.2, we only need to combine Theorem 2.8 and the coprime case of Theorem 1.1 (i.e., $h=1$ ), with the following result, for a proof of which we refer to either [Biswas et al. 2010] or [Schaffhauser 2012].

Proposition 2.10. Let $(X, \sigma)$ be a compact connected real Riemann surface and let $\mathcal{E}$ be a stable holomorphic vector bundle over $X$ satisfying $\overline{\sigma^{*} \mathcal{E}} \simeq \mathcal{E}$. Then $\mathcal{E}$ is either real or quaternionic and cannot be both. Moreover, two different real or quaternionic structures on $\mathcal{E}$ are conjugate by a holomorphic automorphism of $\mathcal{E}$.

Note that it is easy to show that two real or two quaternionic structures on $\mathcal{E}$ differ by a holomorphic automorphism $e^{i \theta} \in S^{1} \subset \mathbb{C}^{*} \simeq \operatorname{Aut}(\mathcal{E})$ but, in order to prove that these two structures $\tau_{1}$ and $\tau_{2}$, say, are conjugate, we need to observe that $\tau_{2}(\cdot)=e^{i \theta} \tau_{1}(\cdot)=e^{i \theta / 2} \tau_{1}\left(e^{-i \theta / 2} \cdot\right)$. Then, to finish the proof of Theorem 1.2, we proceed as follows:

Proof of Theorem 1.2. Recall that $X$ here has genus 1. If $X^{\sigma} \neq \varnothing$, quaternionic vector bundles must have even rank and degree by Theorem 2.5, so, by Proposition 2.10, points of $\mathcal{M}_{X}(r, d)^{\sigma}$ correspond in this case to real isomorphism classes of geometrically stable real vector bundles of rank $r$ and degree $d$. By Theorem 1.1, one indeed has $\mathcal{M}_{X}(r, d)^{\sigma} \simeq\left(\operatorname{Pic}_{X}^{d}\right)^{\sigma} \simeq X^{\sigma}$ in this case. Moreover, since the diffeomorphism $\mathcal{M}_{X}(r, d)^{\sigma} \simeq\left(\operatorname{Pic}_{X}^{d}\right)^{\sigma}$ is provided by the determinant map, the connected components of $\mathcal{M}_{X}(r, d)^{\sigma}$, or equivalently of $\left(\mathrm{Pic}_{X}^{d}\right)^{\sigma}$ are indeed distinguished by the first Stiefel-Whitney class of the real part of the real bundles that they parametrize, as in Theorem 2.8. If now $X^{\sigma}=\varnothing$, then by Theorem 2.5, real and quaternionic vector bundles must have even degree and we can again use Theorem 2.8 to conclude: note that since the diffeomorphism $\mathcal{M}_{X}(r, d)^{\sigma} \simeq\left(\operatorname{Pic}_{X}^{d}\right)^{\sigma}$ is provided by the determinant map, when $d$ is even $r$ must be odd (because $r$ is assumed to be coprime to $d$ ), so the determinant indeed takes real vector bundles to real line bundles and quaternionic vector bundles to quaternionic line bundles.

Had we not assumed $r \wedge d=1$, then the situation would have been more complicated to analyze, because the determinant of a quaternionic vector bundle of even rank is a real line bundle and also because, when $h=r \wedge d$ is even, the real space $\operatorname{Sym}^{h}(X)$ may have real points even if $X$ does not (points of the form $\left[x_{i}, \sigma\left(x_{i}\right)\right]_{1 \leq i \leq h / 2}$ for $\left.x_{i} \in X\right)$.

2E. Real vector bundles of fixed determinant. Let us now consider spaces of vector bundles of fixed determinant. By [Tu 1993, Theorem 3], one has, for any 
$\mathcal{L} \in \operatorname{Pic}_{X}^{d}, \mathcal{M}_{X}(r, \mathcal{L}):=\operatorname{det}^{-1}(\{\mathcal{L}\}) \simeq_{\mathbb{C}} \mathbb{P}_{\mathbb{C}}^{h-1}$ where $d=\operatorname{deg}(\mathcal{L})$ and $h=r \wedge d$. This is proved in the following way: under the identification $\mathcal{M}_{X}(r, d) \simeq_{\mathbb{C}} \operatorname{Sym}^{h}(X)$, there is a commutative diagram

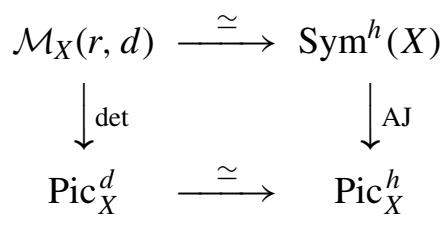

where

$$
\mathrm{AJ}: \operatorname{Sym}^{h}(X) \rightarrow \operatorname{Pic}_{X}^{h}, \quad\left(x_{1}, \ldots, x_{h}\right) \mapsto \mathcal{L}\left(x_{1}+\cdots+x_{h}\right)
$$

is the Abel-Jacobi map (taking a finite family of points $\left(x_{1}, \ldots, x_{h}\right)$ to the line bundle associated to the divisor $\left.x_{1}+\cdots+x_{h}\right)$ and the fiber of the Abel-Jacobi map above a holomorphic line bundle $\mathcal{L}$ of degree $h$ is the projective space $\mathbb{P}\left(H^{0}(X, \mathcal{L})\right)$ which, since $\operatorname{deg}(\mathcal{L})=h \geq 1$ and $X$ has genus 1 , is isomorphic to $\mathbb{P}_{\mathbb{C}}^{h-1}$. Evidently, the same proof will work over $\mathbb{R}$ whenever we can identify $\operatorname{Pic}_{X}^{d}$ and $\operatorname{Pic}_{X}^{h}$ as real varieties, which happens in particular when $X^{\sigma} \neq \varnothing$.

Theorem 2.11. Let $\left(X, x_{0}, \sigma\right)$ be a real elliptic curve satisfying $X^{\sigma} \neq \varnothing$ and let $\mathcal{L}$ be a real line bundle of degree $d$ on $X$. Then, for all $r \geq 1$,

$$
\mathcal{M}_{X}(r, \mathcal{L}) \simeq_{\mathbb{R}} \mathbb{P}_{\mathbb{R}}^{h-1},
$$

where $h=r \wedge d$.

Proof. When $X^{\sigma} \neq \varnothing$, we can choose $x_{0} \in X^{\sigma}$ and use Theorem 2.1 to identify all Picard varieties Pic $_{X}^{k}$ over $\mathbb{R}$, then reproduce Tu's proof recalled above.

\section{Indecomposable vector bundles}

3A. Indecomposable vector bundles over a complex elliptic curve. As recalled in the introduction, a theorem of Grothendieck [1957] shows that any holomorphic vector bundle on $\mathbb{C P}^{1}$ is isomorphic to a direct sum of holomorphic line bundles, and this can be easily recast in modern perspective by using the notions of stability and semistability of vector bundles over curves, introduced by Mumford [1963] and first studied by himself and Seshadri [1967]: the moduli variety of semistable vector bundles of rank $r$ and degree $d$ over $\mathbb{C P}^{1}$ is a single point if $r$ divides $d$ and is empty otherwise. As for vector bundles over a complex elliptic curve, the study was initiated by Atiyah [1957b], thus at a time when the notion of stability was not yet available. Rather, Atiyah's starting point is the notion of an indecomposable vector bundle: a holomorphic vector bundle $\mathcal{E}$ over a complex curve $X$ is said to be indecomposable if it is not isomorphic to a direct sum of nontrivial holomorphic bundles. In the present paper, we shall denote by $\mathcal{I}_{X}(r, d)$ 
the set of isomorphism classes of indecomposable vector bundles of rank $r$ and degree $d$. It is immediate from the definition that a holomorphic vector bundle is a direct sum of indecomposable ones. Moreover, one has the following result, which is a consequence of the categorical Krull-Schmidt theorem, also due to Atiyah, showing that the decomposition of a bundle into indecomposable ones is essentially unique.

Proposition 3.1 [Atiyah 1956, Theorem 3]. Let X be a compact connected complex analytic manifold. Any holomorphic vector bundle $\mathcal{E}$ over $X$ is isomorphic to a direct sum $\mathcal{E}_{1} \oplus \cdots \oplus \mathcal{E}_{k}$ of indecomposable vector bundles. If one also has $\mathcal{E} \simeq \mathcal{E}_{1}^{\prime} \oplus \cdots \oplus \mathcal{E}_{l}^{\prime}$, then $l=k$ and there exists a permutation $\sigma$ of the indices such that $\mathcal{E}_{\sigma(i)}^{\prime} \simeq \mathcal{E}_{i}$.

Going back to the case of a compact, connected Riemann surface $X$ of genus 1 , Atiyah completely describes all indecomposable vector bundles on $X$. He first shows [op. cit., Theorem 5] the existence, for any $h \geq 1$, of a unique (isomorphism class of) indecomposable vector bundle $F_{h}$ of rank $h$ and degree 0 such that

$$
\operatorname{dim} H^{0}\left(X ; F_{h}\right)=1 .
$$

As a matter of fact, this is the only vector bundle of rank $h$ and degree 0 over $X$ with a nonzero space of sections. Let us call $F_{h}$ the Atiyah bundle of rank $h$ and degree 0 . The construction of $F_{h}$ is by induction, starting from $F_{1}=\mathcal{O}_{X}$, the trivial line bundle over $X$, and showing the existence and uniqueness of an extension of the form

$$
0 \rightarrow \mathcal{O}_{X} \rightarrow F_{h} \rightarrow F_{h-1} \rightarrow 0 .
$$

In particular, $\operatorname{det}\left(F_{h}\right)=\mathcal{O}_{X}$. Moreover, Since $F_{h}$ is the unique indecomposable vector bundle with nonzero space of sections, one has [op. cit., Corollary 1]:

$$
F_{h}^{*} \simeq F_{h} .
$$

Note that $F_{h}$ is an extension of semistable bundles so it is semistable. The associated polystable bundle is the trivial bundle of rank $h$, which is not isomorphic to $F_{h}$ (in particular, $F_{h}$ is not itself polystable).

Atiyah then shows that any indecomposable vector bundle $\mathcal{E}$ of rank $h$ and degree 0 is isomorphic to $F_{h} \otimes \mathcal{L}$ for a line bundle $\mathcal{L}$ of degree 0 which is unique up to isomorphism [op. cit., Theorem 5(ii)]. Since it follows from the construction of $F_{h}$ recalled in (3-2) that $\operatorname{det}\left(F_{h}\right)=\mathcal{O}_{X}$, one has $\operatorname{det} \mathcal{E}=\mathcal{L}^{h}$. This sets up a bijection

$$
\operatorname{Pic}_{X}^{0} \rightarrow \mathcal{I}_{X}(h, 0), \quad \mathcal{L} \mapsto F_{h} \otimes \mathcal{L} .
$$

Note that the map (3-4) is just the identity map if $h=1$. Atiyah then uses a marked point $x_{0} \in X$ to further identify $\operatorname{Pic}_{X}^{0}$ with $X$. In particular, the set $\mathcal{I}_{X}(h, 0)$ inherits a natural structure of complex analytic manifold of dimension 1 . 
The next step in Atiyah's characterization of indecomposable vector bundles is to consider the case of vector bundles of nonvanishing degree. He shows [op. cit., Theorem 6] that, associated to the choice of a marked point $x_{0} \in X$, there is, for all $r$ and $d$, a unique bijection (subject to certain conditions)

$$
\alpha_{r, d}^{x_{0}}: \mathcal{I}_{X}(r \wedge d, 0) \rightarrow \mathcal{I}_{X}(r, d)
$$

between the set of isomorphism classes of indecomposable vector bundles of rank $h:=r \wedge d$ and degree 0 and the set of isomorphism classes of indecomposable vector bundles of rank $r$ and degree $d$. As a consequence, Atiyah can define a canonical indecomposable vector bundle of rank $r$ and degree $d$, namely

$$
F_{x_{0}}(r, d):=\alpha_{r, d}^{x_{0}}\left(F_{r \wedge d}\right),
$$

where $F_{r \wedge d}$ is the indecomposable vector bundle of rank $r \wedge d$ and degree 0 whose construction was recalled in (3-2). We will call the bundle $F_{x_{0}}(r, d)$ the Atiyah bundle of rank $r$ and degree $d$ (in particular $F_{x_{0}}(r, 0)=F_{r}$ ). Atiyah then obtains the following description of indecomposable vector bundles:

Theorem 3.2 [Atiyah 1957b, Theorem 10]. Set $h=r \wedge d, r^{\prime}=r / h$, and $d^{\prime}=d / h$. Then every indecomposable vector bundle of rank $r$ and degree $d$ is isomorphic to a bundle of the form $F_{x_{0}}(r, d) \otimes \mathcal{L}$ where $\mathcal{L}$ is a line bundle of degree 0 . Moreover, $F_{x_{0}}(r, d) \otimes \mathcal{L} \simeq F_{x_{0}}(r, d) \otimes \mathcal{L}^{\prime}$ if and only if $\left(\mathcal{L}^{\prime} \otimes \mathcal{L}^{-1}\right)^{r^{\prime}} \simeq \mathcal{O}_{X}$.

Thus, as a generalization to (3-4), Theorem 3.2 shows that there is a surjective map $\operatorname{Pic}_{X}^{0} \rightarrow \mathcal{I}_{X}(r, d)$, whose fiber is isomorphic to the group $T_{r^{\prime}}$ of $r^{\prime}$-torsion elements in $\mathrm{Pic}_{X}^{0}$. This in particular induces a bijection between the Riemann surface $\operatorname{Pic}_{X}^{0} / T_{r^{\prime}} \simeq \operatorname{Pic}_{X}^{0} \simeq X$ and the set $\mathcal{I}_{X}(r, d)$ for all $r$ and $d$, and the set $\mathcal{I}_{X}(r, d)$ inherits in this way a natural structure of complex analytic manifold of dimension 1 .

3B. Relation to semistable and stable bundles. It is immediate to prove that stable bundles (over a curve of arbitrary genus) are indecomposable. Moreover, over an elliptic curve, we have the following result, proved in Tu's paper:

Theorem 3.3 [Tu 1993, Appendix A]. Every indecomposable vector bundle of rank $r$ and degree $d$ over a complex elliptic curve is semistable. It is stable if and only if $r \wedge d=1$.

In particular, the Atiyah bundles $F_{x_{0}}(r, d)$ are semistable (and stable if and only if $r \wedge d=1$ ) and, by Proposition 3.1, every holomorphic vector bundle over a complex elliptic curve is isomorphic to a direct sum of semistable bundles. Next, there is a very important relation between indecomposable vector bundles and stable vector bundles, which will be useful in the next section. 
Theorem 3.4 (Atiyah-Tu). Set $h=r \wedge d, r^{\prime}=r / h$, and $d^{\prime}=d / h$. Then the map

$$
\mathcal{M}_{X}\left(r^{\prime}, d^{\prime}\right) \rightarrow \mathcal{I}_{X}(r, d), \quad \mathcal{E}^{\prime} \mapsto \mathcal{E}^{\prime} \otimes F_{h}
$$

is a bijection: any indecomposable vector bundle of rank $r$ and degree $d$ is isomorphic to a bundle of the form $\mathcal{E}^{\prime} \otimes F_{h}$ where $\mathcal{E}^{\prime}$ is a stable vector bundle of rank $r^{\prime}$ and degree $d^{\prime}$, unique up to isomorphism, and $F_{h}$ is the Atiyah bundle of rank $h$ and degree 0.

In particular, $\mathcal{I}_{X}(r, d)$ inherits in this way a structure of complex analytic manifold of dimension $r^{\prime} \wedge d^{\prime}=1$. This result, which generalizes (3-4) in a different direction than Theorem 3.2, can be deduced from Atiyah and Tu's papers but we give a proof below for the sake of completeness. It is based on the following lemma.

Lemma 3.5 [Atiyah 1957b, Lemma 24]. The Atiyah bundles $F_{x_{0}}(r, d)$ and $F_{x_{0}}\left(r^{\prime}, d^{\prime}\right)$ are related in the following way:

$$
F_{x_{0}}(r, d) \simeq F_{x_{0}}\left(r^{\prime}, d^{\prime}\right) \otimes F_{h} .
$$

Proof of Theorem 3.4. Let $\mathcal{E} \in \mathcal{I}_{X}(r, d)$. By Theorem 3.2, there exists a line bundle $\mathcal{L}$ of degree 0 such that $\mathcal{E} \simeq F_{x_{0}}(r, d) \otimes \mathcal{L}$. By Lemma 3.5, $F_{x_{0}}(r, d) \simeq$ $F_{x_{0}}\left(r^{\prime}, d^{\prime}\right) \otimes F_{h}$. Since $r^{\prime} \wedge d^{\prime}=1$, Theorem 3.3 shows that $F_{x_{0}}\left(r^{\prime}, d^{\prime}\right)$, hence also $\mathcal{E}^{\prime}:=F_{x_{0}}\left(r^{\prime}, d^{\prime}\right) \otimes \mathcal{L}$, are stable bundles of rank $r^{\prime}$ and degree $d^{\prime}$. And one has indeed $\mathcal{E} \simeq \mathcal{E}^{\prime} \otimes F_{h}$. Let now $\mathcal{E}^{\prime}$ and $\mathcal{E}^{\prime \prime}$ be two stable bundles of rank $r^{\prime}$ and degree $d^{\prime}$ such that $\mathcal{E}^{\prime} \otimes F_{h} \simeq \mathcal{E}^{\prime \prime} \otimes F_{h}$. Since stable bundles are indecomposable, Theorem 3.2 shows the existence of two line bundles $\mathcal{L}^{\prime}$ and $\mathcal{L}^{\prime \prime}$ of degree 0 such that $\mathcal{E}^{\prime} \simeq F_{x_{0}}\left(r^{\prime}, d^{\prime}\right) \otimes \mathcal{L}^{\prime}$ and $\mathcal{E}^{\prime \prime} \simeq F_{x_{0}}\left(r^{\prime}, d^{\prime}\right) \otimes \mathcal{L}^{\prime \prime}$. Tensoring by $F_{h}$ and applying Lemma 3.5, we obtain that $F_{x_{0}}(r, d) \otimes \mathcal{L}^{\prime} \simeq F_{x_{0}}(r, d) \otimes \mathcal{L}^{\prime \prime}$, which, again by Theorem 3.2, implies that $\mathcal{L}^{\prime}$ and $\mathcal{L}^{\prime \prime}$ differ by an $r^{\prime}$-torsion point of $\mathrm{Pic}_{X}^{0}$. But then a final application of Theorem 3.2 shows that $F_{x_{0}}\left(r^{\prime}, d^{\prime}\right) \otimes \mathcal{L}^{\prime} \simeq F_{x_{0}}\left(r^{\prime}, d^{\prime}\right) \otimes \mathcal{L}^{\prime \prime}$, i.e., $\mathcal{E}^{\prime} \simeq \mathcal{E}^{\prime \prime}$.

Thus, the complex variety $\mathcal{I}_{X}(r, d) \simeq \mathcal{M}_{X}\left(r^{\prime}, d^{\prime}\right) \simeq X$ is a 1-dimensional subvariety of the $h$-dimensional moduli variety $\mathcal{M}_{X}(r, d) \simeq \operatorname{Sym}^{h}(X)$, and these two nonsingular varieties coincide exactly when $r$ and $d$ are coprime. More explicitly, under the identifications $\mathcal{I}_{X}(r, d) \simeq X$ and $\mathcal{M}_{X}(r, d) \simeq \operatorname{Sym}^{h}(X)$, the inclusion map $\mathcal{I}_{X}(r, d) \hookrightarrow \mathcal{M}_{X}(r, d)$, implicit in Theorem 3.3, is simply the diagonal map

$$
X \rightarrow \operatorname{Sym}^{h}(X), \quad x \mapsto[x, \ldots, x]
$$

and it commutes to the determinant map, the latter being, on $\operatorname{Sym}^{h}(X)$, just the Abel-Jacobi map $\left[x_{1}, \ldots, x_{h}\right] \mapsto x_{1}+\cdots+x_{h}$; see [Tu 1993, Theorem 2].

3C. Indecomposable vector bundles over a real elliptic curve. Over a real elliptic curve, the description of indecomposable vector bundles is a bit more complicated than in the complex case, because the Atiyah map $\alpha_{r, d}^{x_{0}}$ defined in (3-5) is not a real 
map unless the point $x_{0}$ is a real point, which excludes the case where $X^{\sigma}=\varnothing$. Of course the case $X^{\sigma} \neq \varnothing$ is already very interesting and if we follow Atiyah's paper in that case, then the Atiyah map $\alpha_{r, d}^{x_{0}}$ is a real map and the Atiyah bundles $F_{x_{0}}(r, d)$ are all real bundles. In particular, the description given by Atiyah of the ring structure of the set of isomorphism class of all vector bundles (namely the way to decompose the tensor product of two Atiyah bundles into a direct sum of Atiyah bundles, see for instance [Tu 1993, Appendix A] for a concise exposition) directly applies to the subring formed by isomorphism classes real bundles. (Note that, in contrast, isomorphism classes of quaternionic bundles do not form a ring, as the tensor product of two quaternionic bundles is a real bundle.) To obtain a description of indecomposable bundles over a real elliptic curve which holds without assuming that the curve has real points, we need to replace the Atiyah isomorphism

$$
\alpha_{r, d}^{x_{0}}: \mathcal{I}_{X}(r \wedge d, 0) \rightarrow \mathcal{I}_{X}(r, d)
$$

(which cannot be a real map when $X^{\sigma}=\varnothing$ ) by the isomorphism $\mathcal{I}_{X}(r, d) \simeq$ $\mathcal{M}_{X}\left(r^{\prime}, d^{\prime}\right)$ of Theorem 3.4 and show that the latter is always a real map. The first step is the following result, about the Atiyah bundle of rank $h$ and degree 0 , whose definition was recalled in (3-2).

Proposition 3.6. Let $(X, \sigma)$ be a real Riemann surface of genus 1 . For any $h \geq 1$, the indecomposable vector bundle $F_{h}$ has a canonical real structure.

Proof. We proceed by induction. Since $X$ is assumed to be real, $\mathcal{O}_{X}$ has a canonical real structure. So, if $h=1$, then $F_{h}$ is canonically real. Assume now that $h>1$ and that $F_{h-1}$ has a fixed real structure. Following again Atiyah [1957a], extensions of the form (3-2) are parametrized by the sheaf cohomology group $H^{1}\left(X ; \operatorname{Hom}_{\mathcal{O}_{X}}\left(F_{h-1} ; \mathcal{O}_{X}\right)\right)=H^{1}\left(X ; F_{h-1}^{*}\right)$. The uniqueness part of the statement in Atiyah's construction above says that this cohomology group is a complex vector space of dimension 1, which, in any case, can be checked by Riemann-Roch using properties (3-1) and (3-3). Indeed, $\operatorname{since} \operatorname{deg}\left(F_{h-1}^{*}\right)=0$ and $X$ is of genus $g=1$, one has

$$
h^{0}\left(F_{h-1}^{*}\right)-h^{1}\left(F_{h-1}^{*}\right)=\operatorname{deg}\left(F_{h-1}^{*}\right)+\left(\operatorname{rk} F_{h-1}^{*}\right)(1-g)=0,
$$

where $h^{i}(\cdot)=\operatorname{dim} H^{i}(X ; \cdot)$, so $h^{1}\left(F_{h-1}^{*}\right)=h^{0}\left(F_{h-1}^{*}\right)=1$. Now, since $X$ and $F_{h-1}$ have real structures, so does $H^{1}\left(X ; F_{h-1}^{*}\right)$ and the fixed point space of that real structure corresponds to isomorphism classes of real extensions of $F_{h-1}$ by $\mathcal{O}_{X}$. Since the fixed point space of the real structure of $H^{1}\left(X ; F_{h-1}^{*}\right)$ is a 1-dimensional real vector space, the real structure of $F_{h}$ is unique up to isomorphism.

Thus, in contrast to Atiyah bundles of nonvanishing degree, $F_{h}$ is always canonically a real bundle. In particular, $\overline{\sigma^{*} F_{h}} \simeq F_{h}$. It is then clear that the isomorphism

$$
\mathcal{M}_{X}\left(r^{\prime}, d^{\prime}\right) \rightarrow \mathcal{I}_{X}(r, d), \quad \mathcal{E}^{\prime} \mapsto \mathcal{E}^{\prime} \otimes F_{h}
$$


is a real map: $\overline{\sigma^{*} \mathcal{E}^{\prime}} \otimes F_{h} \simeq \overline{\sigma^{*} \mathcal{E}^{\prime}} \otimes \overline{\sigma^{*} F_{h}} \simeq \overline{\sigma^{*}\left(\mathcal{E}^{\prime} \otimes F_{h}\right)}$, which readily implies Theorem 1.3. Moreover, one can make the following observation:

Proposition 3.7. Let $\mathcal{E}$ be an indecomposable vector bundle of rank $r$ and degree $d$ over the real elliptic curve $\left(X, x_{0}, \sigma\right)$ and assume that $\overline{\sigma^{*} \mathcal{E}} \simeq \mathcal{E}$. Then $\mathcal{E}$ admits either a real or a quaternionic structure.

Proof. By Theorem 3.4, we can write $\mathcal{E} \simeq \mathcal{E}^{\prime} \otimes F_{h}$, with $\mathcal{E}^{\prime}$ stable. Therefore,

$$
\overline{\sigma^{*} \mathcal{E}} \simeq \overline{\sigma^{*}\left(\mathcal{E}^{\prime} \otimes F_{h}\right)} \simeq \overline{\sigma^{*} \mathcal{E}^{\prime}} \otimes \overline{\sigma^{*} F_{h}} \simeq \overline{\sigma^{*} \mathcal{E}^{\prime}} \otimes F_{h} .
$$

The assumption $\overline{\sigma^{*} \mathcal{E}} \simeq \mathcal{E}$ then translates to $\overline{\sigma^{*} \mathcal{E}^{\prime}} \otimes F_{h} \simeq \mathcal{E}^{\prime} \otimes F_{h}$ which, since the map from Theorem 3.4 is a bijection, shows that $\overline{\sigma^{*} \mathcal{E}^{\prime}} \simeq \mathcal{E}^{\prime}$. As $\mathcal{E}^{\prime}$ is stable, the fact that $\mathcal{E}^{\prime}$ admits a real or quaternionic structure $\tau^{\prime}$ follows from Proposition 2.10. If $\tau_{h}$ denotes the real structure of $F_{h}$, we then have that $\tau^{\prime} \otimes \tau_{h}$ is a real or quaternionic structure on $\mathcal{E}$, depending on whether $\tau^{\prime}$ is real or quaternionic.

\section{Acknowledgments}

The authors thank the Institute of Mathematical Sciences of the National University of Singapore, for hospitality while the work was carried out. Biswas is supported by the J. C. Bose Fellowship. Schaffhauser acknowledges the support from U.S. National Science Foundation grants DMS 1107452, 1107263, 1107367 "RNMS: GEometric structures And Representation varieties" (the GEAR Network). Thanks also go to the referee for a careful reading of the paper and for suggesting the reference [Baird 2014].

\section{References}

[Atiyah 1956] M. F. Atiyah, "On the Krull-Schmidt theorem with application to sheaves", Bull. Soc. Math. France 84 (1956), 307-317. MR 0086358 Zbl 0072.18101

[Atiyah 1957a] M. F. Atiyah, "Complex analytic connections in fibre bundles", Trans. Amer. Math. Soc. 85 (1957), 181-207. MR 0086359 Zbl 0078.16002

[Atiyah 1957b] M. F. Atiyah, "Vector bundles over an elliptic curve", Proc. London Math. Soc. (3) 7 (1957), 414-452. MR 0131423 Zbl 0084.17305

[Baird 2014] T. Baird, "Moduli spaces of vector bundles over a real curve: $\mathbb{Z} / 2$-Betti numbers", Canad. J. Math. 66:5 (2014), 961-992. MR 3251762 Zbl 1300.32019

[Biswas et al. 2010] I. Biswas, J. Huisman, and J. Hurtubise, "The moduli space of stable vector bundles over a real algebraic curve", Math. Ann. 347:1 (2010), 201-233. MR 2593289 Zbl 1195.14048

[Friedman et al. 1998] R. Friedman, J. W. Morgan, and E. Witten, "Principal G-bundles over elliptic curves", Math. Res. Lett. 5:1-2 (1998), 97-118. MR 1618343 Zbl 0937.14019

[Gross and Harris 1981] B. H. Gross and J. Harris, "Real algebraic curves", Ann. Sci. École Norm. Sup. (4) 14:2 (1981), 157-182. MR 631748 Zbl 0533.14011

[Grothendieck 1957] A. Grothendieck, "Sur la classification des fibrés holomorphes sur la sphère de Riemann”, Amer. J. Math. 79 (1957), 121-138. MR 0087176 Zbl 0079.17001 
[Laszlo 1998] Y. Laszlo, “About G-bundles over elliptic curves”, Ann. Inst. Fourier (Grenoble) 48:2 (1998), 413-424. MR 1625614 Zbl 0901.14019

[Liu and Schaffhauser 2013] C.-C. M. Liu and F. Schaffhauser, "The Yang-Mills equations over Klein surfaces”, J. Topol. 6:3 (2013), 569-643. MR 3100884 Zbl 1288.14022

[Looijenga 1976] E. Looijenga, "Root systems and elliptic curves", Invent. Math. 38:1 (1976), 17-32. MR 0466134 Zbl 0358.17016

[Mumford 1963] D. Mumford, "Projective invariants of projective structures and applications", pp. 526-530 in Proceedings of the International Congress of Mathematicians (Stockholm, 1962), Institut Mittag-Leffler, Djursholm, 1963. MR 0175899 Zbl 0154.20702

[Narasimhan and Seshadri 1965] M. S. Narasimhan and C. S. Seshadri, "Stable and unitary vector bundles on a compact Riemann surface", Ann. of Math. (2) 82 (1965), 540-567. MR 0184252 Zbl 0171.04803

[Schaffhauser 2012] F. Schaffhauser, "Real points of coarse moduli schemes of vector bundles on a real algebraic curve", J. Symplectic Geom. 10:4 (2012), 503-534. MR 2982021 Zbl 06141803

[Seshadri 1967] C. S. Seshadri, "Space of unitary vector bundles on a compact Riemann surface", Ann. of Math. (2) 85 (1967), 303-336. MR 0233371 Zbl 0173.23001

[Tu 1993] L. W. Tu, "Semistable bundles over an elliptic curve", Adv. Math. 98:1 (1993), 1-26. MR 1212625 Zbl 0786.14021

Received September 21, 2015. Revised December 28, 2015.

INDRANIL BISWAS

SCHOOL OF MATHEMATICS

TATA Institute of FundaMENTAL RESEARCH

HOMI BHABHA ROAD

MUMBAI 400005

INDIA

indranil@math.tifr.res.in

FLORENT SCHAFFHAUSER

DEPARTAMENTo de Matemáticas

UNIVERSIDAD DE LOS ANDES

BOGOTÁ

Colombia

florent@uniandes.edu.co 


\title{
PACIFIC JOURNAL OF MATHEMATICS
}

Founded in 1951 by E. F. Beckenbach (1906-1982) and F. Wolf (1904-1989)

$$
\text { msp.org/pjm }
$$

\section{EDITORS}

\author{
Don Blasius (Managing Editor) \\ Department of Mathematics \\ University of California \\ Los Angeles, CA 90095-1555 \\ blasius@math.ucla.edu
}

\author{
Paul Balmer \\ Department of Mathematics \\ University of California \\ Los Angeles, CA 90095-1555 \\ balmer@math.ucla.edu \\ Robert Finn \\ Department of Mathematics \\ Stanford University \\ Stanford, CA 94305-2125 \\ finn@math.stanford.edu \\ Sorin Popa \\ Department of Mathematics \\ University of California \\ Los Angeles, CA 90095-1555 \\ popa@math.ucla.edu
}

\author{
Vyjayanthi Chari \\ Department of Mathematics \\ University of California \\ Riverside, CA 92521-0135 \\ chari@math.ucr.edu \\ Kefeng Liu \\ Department of Mathematics \\ University of California \\ Los Angeles, CA 90095-1555 \\ liu@math.ucla.edu \\ Igor Pak \\ Department of Mathematics \\ University of California \\ Los Angeles, CA 90095-1555 \\ pak.pjm@gmail.com \\ Paul Yang \\ Department of Mathematics \\ Princeton University \\ Princeton NJ 08544-1000 \\ yang@math.princeton.edu
}

\section{PRODUCTION}

Silvio Levy, Scientific Editor, production@msp.org

\section{SUPPORTING INSTITUTIONS}

ACADEMIA SINICA, TAIPEI

CALIFORNIA INST. OF TECHNOLOGY

STANFORD UNIVERSITY

UNIV. OF BRITISH COLUMBIA

UNIV. OF CALIFORNIA, BERKELEY

UNIV. OF CALIFORNIA, DAVIS

UNIV. OF CALIFORNIA, LOS ANGELES

UNIV. OF CALIFORNIA, RIVERSIDE

UNIV. OF CALIFORNIA, SAN DIEGO

UNIV. OF CALIF., SANTA BARBARA
KEIO UNIVERSITY

MATH. SCIENCES RESEARCH INSTITUTE

NEW MEXICO STATE UNIV.

OREGON STATE UNIV.
Daryl Cooper

Department of Mathematics

University of California

Santa Barbara, CA 93106-3080 cooper@math.ucsb.edu

Jiang-Hua Lu

Department of Mathematics

The University of Hong Kong

Pokfulam Rd., Hong Kong

jhlu@maths.hku.hk

$$
\text { Jie Qing }
$$

Department of Mathematics

University of California

Santa Cruz, CA 95064

qing@cats.ucsc.edu

\author{
UNIV. OF CALIF., SANTA CRUZ \\ UNIV. OF MONTANA \\ UNIV. OF OREGON \\ UNIV. OF SOUTHERN CALIFORNIA \\ UNIV. OF UTAH \\ UNIV. OF WASHINGTON \\ WASHINGTON STATE UNIVERSITY
}

These supporting institutions contribute to the cost of publication of this Journal, but they are not owners or publishers and have no responsibility for its contents or policies.

See inside back cover or msp.org/pjm for submission instructions.

The subscription price for 2016 is US \$/year for the electronic version, and \$/year for print and electronic.

Subscriptions, requests for back issues and changes of subscriber address should be sent to Pacific Journal of Mathematics, P.O. Box 4163, Berkeley, CA 94704-0163, U.S.A. The Pacific Journal of Mathematics is indexed by Mathematical Reviews, Zentralblatt MATH, PASCAL CNRS Index, Referativnyi Zhurnal, Current Mathematical Publications and Web of Knowledge (Science Citation Index).

The Pacific Journal of Mathematics (ISSN 0030-8730) at the University of California, c/o Department of Mathematics, 798 Evans Hall \#3840, Berkeley, CA 94720-3840, is published twelve times a year. Periodical rate postage paid at Berkeley, CA 94704, and additional mailing offices. POSTMASTER: send address changes to Pacific Journal of Mathematics, P.O. Box 4163, Berkeley, CA 94704-0163.

PJM peer review and production are managed by EditFLOW ${ }^{\circledR}$ from Mathematical Sciences Publishers.

PUBLISHED BY

\section{I. mathematical sciences publishers}

nonprofit scientific publishing

http://msp.org/

(C) 2016 Mathematical Sciences Publishers 


\section{PACIFIC JOURNAL OF MATHEMATICS}

Volume $283 \quad$ No. $1 \quad$ July 2016

A New family of simple $\mathfrak{g l}_{2 n}(\mathbb{C})$-modules

JONATHAN NILSSON

Derived categories of representations of small categories over commutative noetherian rings

BENJAMIN ANTIEAU and GREg STEVENSON

Vector bundles over a real elliptic curve

INDRANIL BISWAS and FLORENT SCHAFFHAUSER

$\mathrm{Q}(\mathrm{N})$-graded Lie superalgebras arising from fermionic-bosonic representations

JIN CHENG

Conjugacy and element-conjugacy of homomorphisms of compact Lie groups

YiNGJUE FANG, GANG HAN and BINYONG SUN

Entire sign-changing solutions with finite energy to the fractional Yamabe equation

DANILO GARRIDO and MoniCa Musso

Calculation of local formal Mellin transforms

ADAM GRAHAM-SQUIRE

The untwisting number of a knot

KENAN INCE

A Plancherel formula for $L^{2}(G / H)$ for almost symmetric subgroups

Bent Ørsted and Birgit SPEH

Multiplicative reduction and the cyclotomic main conjecture for $\mathrm{GL}_{2}$

CHRISTOPHER SKINNER

Commensurators of solvable $S$-arithmetic groups

\section{DANIEL STUDENMUND}

Gerstenhaber brackets on Hochschild cohomology of quantum symmetric algebras and their group extensions

SARAH WITHERSPOON and GUODONG ZHOU 